\title{
Simple Oscillator with Enlarged Tunability Range Based on ECCII and VGA Utilizing Commercially Available Analog Multiplier
}

\author{
Roman Sotner ${ }^{1}$, Jan Jerabek ${ }^{2}$, Norbert Herencsar ${ }^{2}$, Jiun-Wei Horng ${ }^{3}$, Kamil Vrba ${ }^{2}$, Tomas Dostal ${ }^{1,4}$ \\ ${ }^{1}$ Dept. of Radio Electronics, Faculty of Electrical Engineering and Communication, Brno University of Technology, \\ Technicka 3082/12, 616 00, Brno, Czech Republic, sotner@feec.vutbr.cz. \\ ${ }^{2}$ Dept. of Telecommunications, Faculty of Electrical Engineering and Communication, Brno University of Technology, \\ Technicka 3082/12, 616 00, Brno, Czech Republic \\ ${ }^{3}$ Dept. of Electronic Engineering, Chung Yuan Christian University, 32023, Chung-Li, Taiwan \\ ${ }^{4}$ Dept. of Technical Studies, College of Polytechnics Jihlava, Tolsteho 16, 586 01, Jihlava, Czech Republic
}

\begin{abstract}
This work presents an example of implementation of electronically controllable features to an originally unsuitable circuit structure of oscillator. Basic structure does not allow any electronic control and has mutually dependent condition of oscillation (CO) and frequency of oscillation (FO) if only values of passive elements are considered as the only way of control. Utilization of electronically controllable current conveyor of second generation (ECCII) brings control of CO independent of FO. Additional application of voltage amplifier with variable gain in both polarities (voltage-mode multiplier) to feedback loop allows also important enlargement of the range of the independent FO control. Moreover, our proposal was tested and confirmed experimentally with commercially available active elements ("Diamond transistor", current-mode multiplier, voltage-mode multiplier) in working range of tens of MHz.
\end{abstract}

Keywords: Current- and voltage-mode multipliers, oscillators, electronic control, mutual dependence of condition and frequency, diamond transistor, variable gain, voltage amplifier.

\section{INTRODUCTION}

Mutual dependence of condition of oscillation ( $\mathrm{CO}$ ) and frequency of oscillation (FO) is a problem of several conceptions of sinusoidal oscillators. This group of circuits has parameters (circuit element values) of FO included also in $\mathrm{CO}$ and vice versa. Typical examples can be found in [1] [2], for example.

Another situation is when control of FO is provided by one parameter separated from others included in $\mathrm{CO}$ relation. Thus, control of FO seems to be really independent of CO. Unfortunately, parameter(s) required for CO control (startup of oscillations and amplitude stabilization during the tuning process) occur in the FO formula. Parameter that is unchangeable only seemingly may have impact on FO (it depends on specific solution and design, whether real frequency is influenced significantly, slightly or even insignificantly). It is significant especially if wideband tuning is required and, at least, stability of FO is affected. Typical examples can be found in [3]-[5].
On the other hand, simple solutions where $\mathrm{CO}$ is independently controllable are also available [6]. However, FO tuning has direct impact on $\mathrm{CO}$ fulfillment (parameter from $\mathrm{FO}$ is present in $\mathrm{CO}$ ) and therefore the independent parameter that keeps $\mathrm{CO}$ fulfilled must be adjusted during the tuning process too. This is also unsuitable.

Some of the previous works discuss also specific solutions with theoretically independent $\mathrm{CO}$ and FO. $\mathrm{CO}$ of such solutions is theoretically always fulfilled if equality of working capacitors and other passive or/and active elements is ensured (for example [7]). Theoretical control of FO without impact on $\mathrm{CO}$ and vice versa is available only if exact matching (equality) of two parameters simultaneously used for FO tuning and CO fulfillment is ensured. In most cases, the same passive elements (parameters of active elements) are included in $\mathrm{CO}$ and also in FO term (for example [7]-[12]) and critical matching of two parameters is required for simultaneous fulfillment of $\mathrm{CO}$ and tuning of FO (typical example: $\mathrm{CO}: g_{\mathrm{m} 1}=g_{\mathrm{m} 2} ; \mathrm{FO}: \sqrt{ }\left(g_{\mathrm{m} 1} g_{\mathrm{m} 2} /\left(C_{1} C_{2}\right)\right)$, see [8], [11], [12], for example). 
Unfortunately, startup of oscillations requires slight inequality of both parameters in $\mathrm{CO}$ relation which can be theoretically preserved during the FO tuning. In addition, $\mathrm{CO}$ driving is required in real situation for amplitude stabilization during the tuning process ([13] and references cited therein) and additional independent parameter suitable for this operation is not available in this kind of solution. Thus, CO and FO are mutually dependent also in this case. This dependence is not so obvious but it is caused by problematic matching (and driving) of two suitable parameters serving for $\mathrm{FO}$ control and $\mathrm{CO}$ fulfillment simultaneously. It is a common problem of very simple solutions having low number of active elements. This drawback is typical also for some of the simple or complex third- or higher-order systems (selected solutions available for example in [14]-[17]).
Some of the above discussed circuits were designed in order to be very simple (minimal number of active and passive elements) and to provide linear control of FO ([10], [12] for example). However, lack of independently controllable parameter for $\mathrm{CO}$ control complicates their practical utilization. Very simple oscillator solutions (up to two simple active elements) usually offer only nonlinear control of FO (if CO and FO are really independent). Some types of the nonlinearly controllable oscillators have also minimal available FO [3]-[5] given by special relation in numerator of FO equation. Some realizations have even more unsuitable equation for FO, where FO control is quite limited even by two borders [4]. Important goal of this paper is to show that slight modification of these simple solutions could bring significant improvements.

Table 1. Comparison of recently published tunable oscillators.

\begin{tabular}{|c|c|c|c|c|c|c|c|c|c|}
\hline 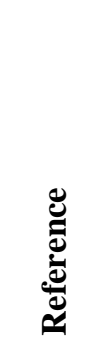 & 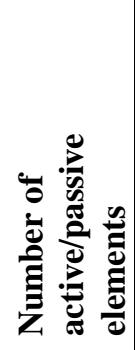 & 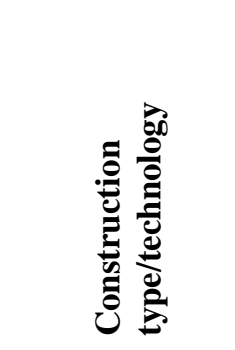 & 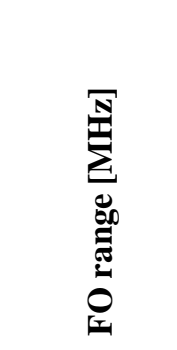 & 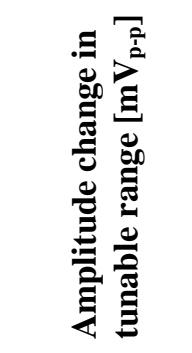 & 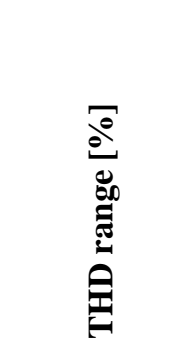 & 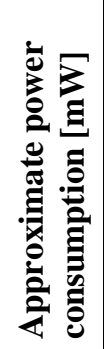 & 总 & 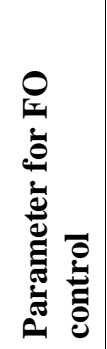 & 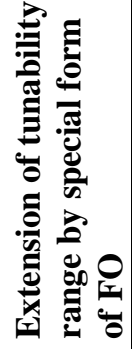 \\
\hline [3] & $3 / 4$ & discrete & $0.6-2.2$ & N/A & $0.4-0.9$ & 250 & $\begin{array}{c}\text { internal } \\
\text { nonlinearity }\end{array}$ & $B$ & No \\
\hline [4] & $2 / 4$ & discrete & $0.3-1.8$ & N/A & $0.3-10$ & 180 & $\begin{array}{c}\text { manual } \\
\text { adjusting }\end{array}$ & $B$ & No \\
\hline [5] & $2 / 3$ & CMOS & $0.6-2.5$ & $350-410$ & 0.8 & N/A & $\begin{array}{c}\text { internal } \\
\text { nonlinearity }\end{array}$ & $g_{\mathrm{m}}$ & No \\
\hline [10] & $1 / 2$ & CMOS & N/A & N/A & 2.9 & N/A & N/A & $R_{\mathrm{X}}$ & No \\
\hline [11] & $3 / 2$ & N/A & N/A & $\mathrm{N} / \mathrm{A}$ & N/A & N/A & N/A & $g_{\mathrm{m}}$ & No \\
\hline [12] & $1 / 2$ & BJT & N/A & N/A & N/A & N/A & $\begin{array}{c}\text { internal } \\
\text { nonlinearity }\end{array}$ & $g_{\mathrm{m}}$ & No \\
\hline [13] & $2 / 5$ & CMOS/discrete & $0.3-8.0$ & $180-240$ & $\begin{array}{c}0.3-5 \\
(0.3-0.5)^{*}\end{array}$ & 450 & $\begin{array}{c}\text { inertial } \\
\text { AGC }\end{array}$ & $B, A$ & No \\
\hline [14] & $2-4 / 2-4$ & CMOS & up to 69.0 & $200-500$ & $0.2-2.8$ & N/A & $\begin{array}{c}\text { inertial } \\
\text { AGC }\end{array}$ & $g_{\mathrm{m}}$ & No \\
\hline [15] & $3 / 3$ & CMOS & $0.1-4.0$ & $\begin{array}{c}500-1700 \\
0-3000\end{array}$ & $>1$ & N/A & el. resistor & $g_{\mathrm{m}}$ & No \\
\hline [16] & $3 / 3$ & CMOS & $0.03-0.09$ & N/A & up to 2.6 & 4 & N/A & $g_{\mathrm{m}}$ & No \\
\hline [17] & $3 / 5$ & CMOS & $4.0-9.0$ & N/A & $0.5-1.8$ & N/A & N/A & $R$ & No \\
\hline [26] & $1 / 4$ & CMOS & $1.3-7.4$ & $\begin{array}{l}50-60 \\
60-90 \\
\end{array}$ & $0.5-3.8$ & $\begin{array}{c}5.8- \\
6.8 \\
\end{array}$ & $\begin{array}{c}\text { inertial } \\
\text { AGC }\end{array}$ & $g_{\mathrm{m}} / R_{\mathrm{X}}$ & No \\
\hline [27] & $2 / 2$ & discrete & $0.5-1.8$ & $\begin{array}{c}600-700 \\
700-750 \\
950-1150\end{array}$ & $0.5-1$ & N/A & $\begin{array}{c}\text { inertial } \\
\text { AGC }\end{array}$ & $g_{\mathrm{m}} / R_{\mathrm{X}}$ & No \\
\hline prop. & $2 / 4$ & discrete & $3.7-27.1$ & $175-205$ & $\begin{array}{c}0.2-3.7 \\
(0.2-1.5)^{*}\end{array}$ & 300 & $\begin{array}{c}\text { inertial } \\
\text { AGC }\end{array}$ & $A$ & Yes \\
\hline
\end{tabular}

*in slightly reduced bandwidth

$B$ - current gain controllable by DC voltage

$A$ - voltage gain controllable by DC voltage

$R \mathrm{X}$ - control of internal resistance of current input terminal by DC bias current

$R$ - passive resistance value change

$g_{\mathrm{m}}-$ transconductance controllable by DC bias current

N/A - not available, not tested 
Detailed qualitative comparison of previously reported related works is given in Table 1. It can be seen that only circuits in ref. [14] have better frequency features. However, in accordance with the results in [14], produced signals significantly change level during the tuning process and the structures with the best performances have more active devices (without considering AGC loop) than the solution presented in this paper. Higher power consumption of discrete active devices utilized in our circuit is the cost for utilization of commercially available devices based on BJT and technology target on great dynamical range and great linearity. It allows production of output signal level in hundreds of $\mathrm{mV}_{\mathrm{p}-\mathrm{p}}$ in comparison to many CMOS solutions (highly nonlinear and with limited output swing to several tens of $\mathrm{mV}$ or $\mu \mathrm{V}$ in many cases) presented in literature. It is worth mentioning that none from the compared and other known solutions in literature utilizes additional tunability extension in nonlinearly tunable oscillators by both polarities of voltage gain available in simple voltage multiplier presented in the frame of the VGA structure used in this oscillator.

Presented example shows that some initially unsuitable solutions (with mutually dependent $\mathrm{CO}$ and FO given by passive elements in many cases) for electronically adjustable applications can be also modified to perform better. This modification consists only of additional feedback loop. Alternate polarity of gain in this loop brings extension of the range of FO control that was originally not possible and similarly simple circuits [3]-[5] have limit at the low corner of FO range (FO cannot be tuned from zero in ideal case).

This paper focuses on implementation of electronic control to a simple type of the second-order harmonic oscillator, where independent (or even electronic) control of $\mathrm{CO}$ and FO is not possible in initial state. However, implementation of controlled current and voltage gains helps to control CO and FO independently. It also directly prepares for amplitude stabilization by a quite simple amplitude automatic gain control circuit (AGC).

The paper is organized as follows: Reasons for this research and improvements in this area of simple oscillators and explanation of situation are given in the introductory section. Next chapter deals briefly with the principle of active elements used in our design. Proposal of the controllable oscillator is discussed in section 3. Real behavior and experiments are given in chapter 4 and finally, concluding remarks are in section 5 .

\section{INTRODUCTION TO THE PRINCIPLE OF USED ACTIVE ELEMENTS}

Basic ideal principles of used active elements are explained in Fig.1. and the following text. The function of electronically controllable current conveyor of second generation (ECCII+) [18]-[20] (Fig.1.a)) is described by quite common and simple equations (in ideal case): $V_{\mathrm{Y}}=V_{\mathrm{X}}$, $I_{\mathrm{Y}}=0, I_{\mathrm{Z}_{+}}=B I_{\mathrm{X}}$. The current gain between $\mathrm{X}$ and $\mathrm{Z}$ ports $(B)$ is adjustable by DC voltage $\left(V_{\mathrm{SETB}}\right)$ and voltage gain (transfer) from $\mathrm{Y}$ to $\mathrm{X}$ port is fixed (equal to 1). Variable
Gain Amplifier (VGA) in Fig.1.b) with gain (A) controllable by DC voltage $V_{\text {SETA }}$ in both polarities was constructed by high-speed voltage-mode four-quadrant multiplier that contains also additional summing point. Detailed connection of multiplier (AD835 in our case) as amplifier with $\pm A$ is shown in Fig.1.c). Transfer function of the amplifier has the form:

$$
\pm A= \pm V_{\text {SETA }}\left(\frac{R_{f 1}+R_{f 2}}{R_{f 2}}\right),
$$

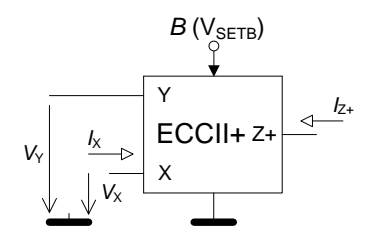

a)

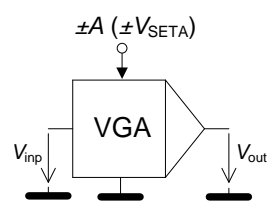

b)

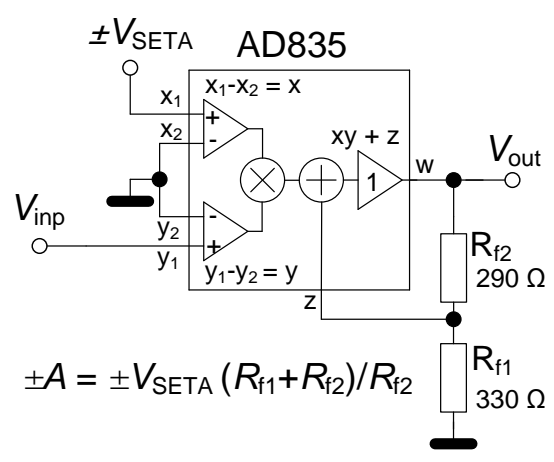

c)

Fig.1. Principles of used active elements: a) ECCII+, b) variable gain amplifier, c) detailed application of multiplier as VGA.

\section{OSCILLATOR PROPOSAL}

Our goal is to not show a fully linearly controllable and multiphase type of oscillator. Instead we want to discuss useful application of the voltage mode multiplier in oscillator structure that is not normally suitable for electronic control. Structure shown in Fig.2. employs ECCII, two resistors and two grounded capacitors. It has no capability of electronic control of FO. When using classical positive current conveyor of second generation (CCII+) [21], [22], $\mathrm{CO}$ and FO are mutually dependent and the solution is not really suitable for practice. In our case, $\mathrm{CO}$ is adjustable by current gain $B$ of the ECCII. In addition, control by passive elements is also not possible due to their mutual appearance in equations for $\mathrm{FO}$ and $\mathrm{CO}$. 


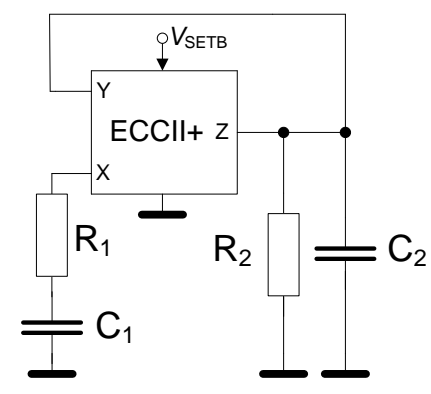

Fig.2. Simple single ECCII-based oscillator.

Characteristic equation of the circuit in Fig.2. has the following form:

$$
s^{2}+\frac{R_{1} C_{1}+R_{2} C_{2}-R_{2} C_{1} B}{R_{1} R_{2} C_{1} C_{2}} s+\frac{1}{R_{1} R_{2} C_{1} C_{2}}=0 .
$$

Initial circuit in Fig.2. is a good starting point for our modification, which consists of insertion of an additional voltage gain $A$ (one active device to the circuit, VGA connected as in Fig.1.c). Resulting circuit has independently adjustable $\mathrm{FO}$ and $\mathrm{CO}$ by electronically controllable parameters. The number of passive elements remains the same, only resistors are floating as is obvious from Fig.3.

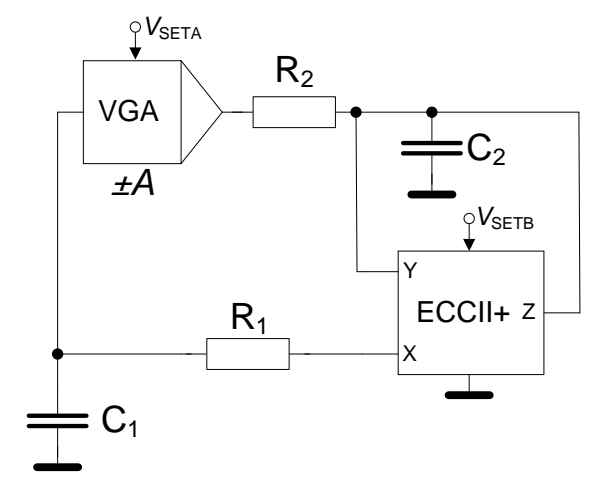

Fig.3. Proposed modification of circuit from Fig.2. to fully and independently controllable oscillator.

Characteristic equation is modified from (2) to:

$$
s^{2}+\frac{R_{1} C_{1}+R_{2} C_{2}-R_{2} C_{1} B}{R_{1} R_{2} C_{1} C_{2}} s+\frac{1 \pm A}{R_{1} R_{2} C_{1} C_{2}}=0
$$

where $\mathrm{CO}$ and $\mathrm{FO}$ are as follows:

$$
\begin{gathered}
B \geq \frac{R_{1} C_{1}+R_{2} C_{2}}{R_{2} C_{1}}, \\
\omega_{0}=\sqrt{\frac{1 \pm A}{R_{1} R_{2} C_{1} C_{2}}}=\sqrt{\frac{1 \pm V_{\text {SETA }}\left(\frac{R_{f 1}+R_{f 2}}{R_{f 2}}\right)}{R_{1} R_{2} C_{1} C_{2}} .}
\end{gathered}
$$

Gain polarity of the voltage amplifier can be considered as positive only $(+A)$. In this case, FO has lower limit given by 1 (for $A=0$ ) in numerator, as we can see from equation (5). Therefore, FO cannot be equal to 0 in ideal case. This fact also reduces frequency range of FO control. Due to root square, FO is not controllable linearly. But this is an obvious problem in such a simple circuit. Utilization of standard VGA, for example VCA810 [23], LMH6505 [24], leads to reduced FO control range. Fortunately, high-speed analog multiplier (AD835 [25]) applied as VGA in our case allows simultaneous change of gain polarity $( \pm A)$, which seems to be very beneficial in this application. It offers enlargement of FO control (below 1 in numerator of (5)) with limit of $A>-1$.

Relation between nodal voltages on both capacitors is:

$$
\frac{V_{C 2}}{V_{C 1}}=\frac{R_{1} A-R_{2} B}{R_{1}-R_{2} B+s C_{2} R_{1} R_{2}} .
$$

It indicates that FO control by $A$ has direct impact on one generated amplitude $\left(V_{\mathrm{C} 2}\right)$ and compensation changes of gain in the structure for CO control (amplitude stabilization) by $B$ also cause impact on phase shift. Therefore, similar simple oscillators are not suitable for tunable multiphase/quadrature signal generation, with some exceptions [26], [27]. Nevertheless, this solution is sufficient for single-output oscillator type (output voltage on $C_{1}$ ) with mutually independent $\mathrm{CO}$ a FO.

\section{REAL BEHAVIOR AND EXPERIMENTAL VERIFICATION}

Real solution of the oscillator from Fig.3. can be formed by commercially available devices as shown in Fig.4. We have to use ECCII- type (EL2082 [28]) and current inverter formed by "Diamond circuit", well-known as "Diamond transistor" (DT) OPA860 [29], because ECCII+ is not directly available. Voltage buffer (subpart of OPA860) connected to the node with $C_{1}$ ensures low-impedance voltage output for measurement purposes. AGC is also implemented in this real solution shown in Fig.4.

Our aim is to design an oscillator operating in range of tens of $\mathrm{MHz}$ (used active elements allow it). Thus, working capacitors and resistors with very low values were selected $\left(C_{1}=C_{2}=C=33 \mathrm{pF}, \quad R_{1}=120+95 \Omega\right.$ and $\left.R_{2}=220 \Omega\right)$, where $R_{1}$ includes internal resistance of terminal X of ECCII $(95 \Omega)$ [28]. High-frequency design requires the best knowledge of all parasitic influences. Non-ideal model of the oscillator with important parasitic features is shown in Fig.5. The most important are parasitic capacitances in nodes, because they have values comparable with working capacitors. Therefore, their impact is substantial and they cause a very large difference between ideal and real FO. They are formed by capacitances of terminals of active elements [25], [28], [29] and also by PCB capacitances and parasitic feedback crosstalks. Thus, we expect them to be $\sim 20 \mathrm{pF}$. Estimated values are noted in Fig.5. (purple color).

FO in influenced (real) case can be calculated as:

$$
\omega_{0}^{\prime}=\sqrt{\frac{A R_{p 1} R_{p 2}+R_{p 1} R_{p 2}+R_{1}^{\prime} R_{2}+R_{p 1} R_{2}-B R_{p 2} R_{2}}{R_{1}^{\prime} R_{2} R_{p 1} R_{p 2} C_{1}^{\prime} C_{2}^{\prime}}}
$$


and real $\mathrm{CO}$ has the form:

$$
B \geq \frac{R_{p 1} R_{p 2}\left(R_{1} C_{1}^{\prime}+R_{2} C_{2}^{\prime}\right)+R_{1} R_{2}\left(R_{p 1} C_{1}^{\prime}+R_{p 2} C_{2}^{\prime}\right)}{R_{2} R_{p 1} R_{p 2} C_{1}^{\prime}} .
$$

CO actually influences FO in real case, see (7). Fortunately, impact of negative term $-B R_{\mathrm{p} 2} R_{2}$ is significantly lower than term $A R_{\mathrm{p} 1} R_{\mathrm{p} 2}$. Therefore, its influence on $\mathrm{FO}$ is significant only for very low $A$ or for large change of $B$ while $A$ is constant (not allowed by AGC).

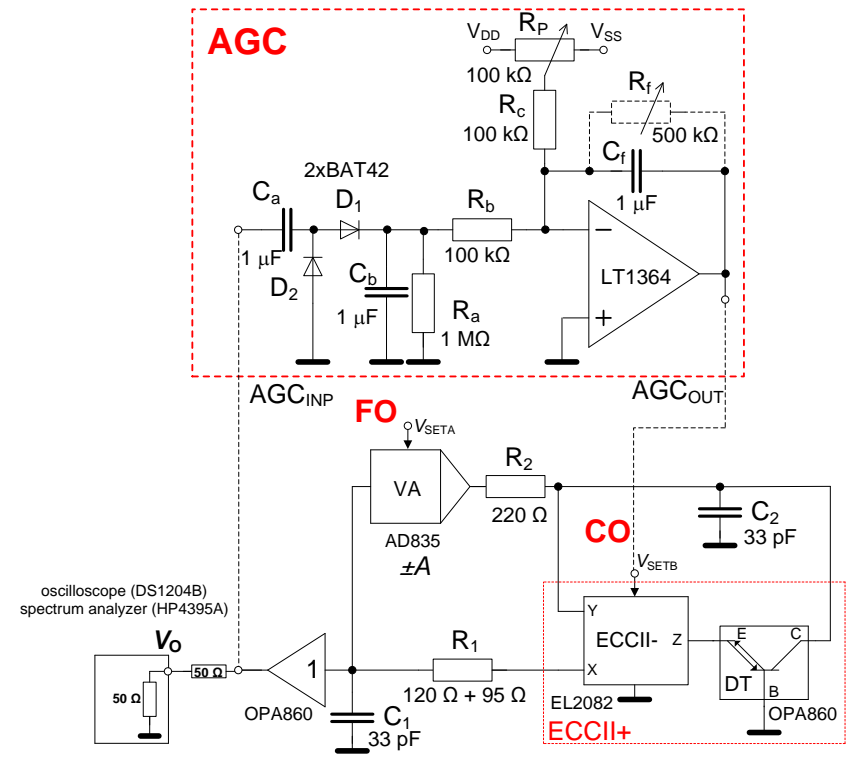

Fig.4. Real circuit for experimental tests.

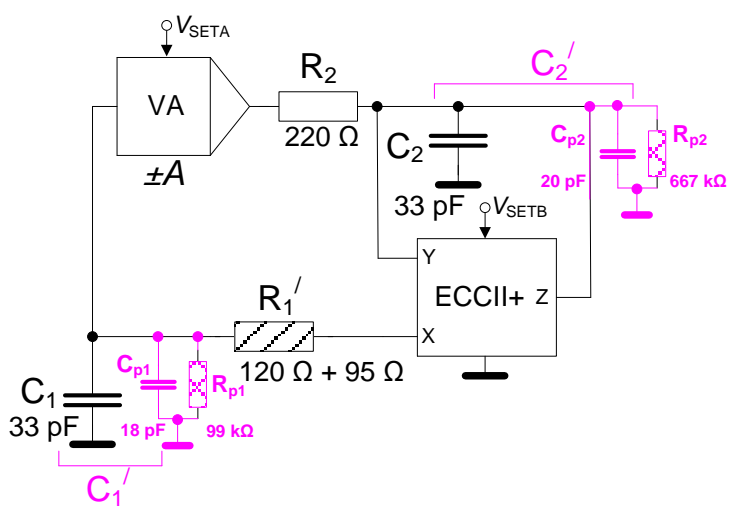

Fig.5. Model for estimation of parasitic influences in the structure of oscillator.

Ideal range of tunability (for discussed ideal values of $R$, $C$ ) and $A$ in range from -0.94 to 3.71 ( $V_{\text {SETA }}$ from -0.44 to $1.74 \mathrm{~V}$ ) was calculated from 5.4 to $48.2 \mathrm{MHz}$. Results are shown in Fig.6. Estimation of FO range, based on analysis of model in Fig.5., was provided from a full representation of real FO (7). It provides range of $\mathrm{FO}$ from 3.3 to $30.6 \mathrm{MHz}$. FO range from 3.7 to $27.1 \mathrm{MHz}$ was obtained from experimental measurements (setup was shown in Fig.4.). Overlying of estimated and measured trace in Fig.6. occurs for $C_{\mathrm{p} 1}=C_{\mathrm{p} 2}=25 \mathrm{pF}$ in (7). If gain $A$ was available only in positive polarity, tunability range would be restricted only to $12-27 \mathrm{MHz}$. Note: We used RIGOL DS1204B oscilloscope and HP4395A spectrum analyzer. Condition of oscillation is ideally fulfilled for $B=2$ (in case of equal $C$ and $R$ as stated above). However, as we can see in Fig.7., $V_{\text {SETB }}$ (practically equal to gain $B$, see [28]) indicates that the gain changes during the tuning process as was explained in [13].

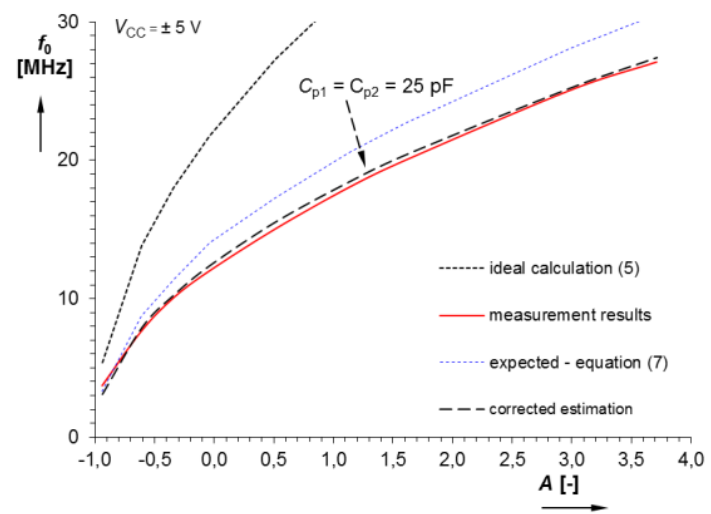

a)

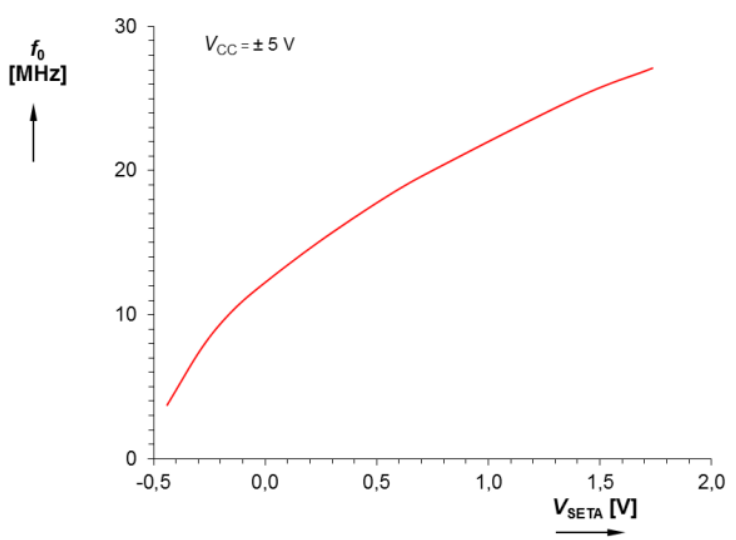

b)

Fig.6. Dependence of FO on: a) voltage gain $A$ - comparison, b) DC control voltage $V_{\text {SETA }}$.

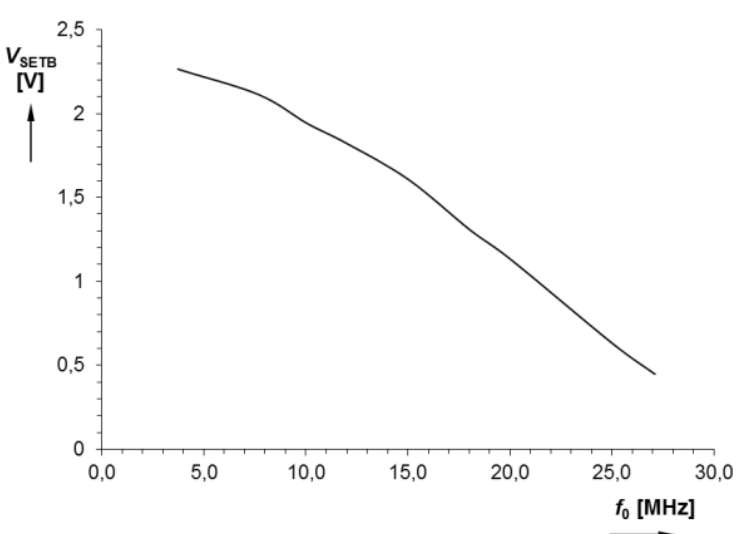

Fig.7. Dependence of $V_{\mathrm{SETB}} \cong B$ (actually $\mathrm{CO}$ ) on FO. 


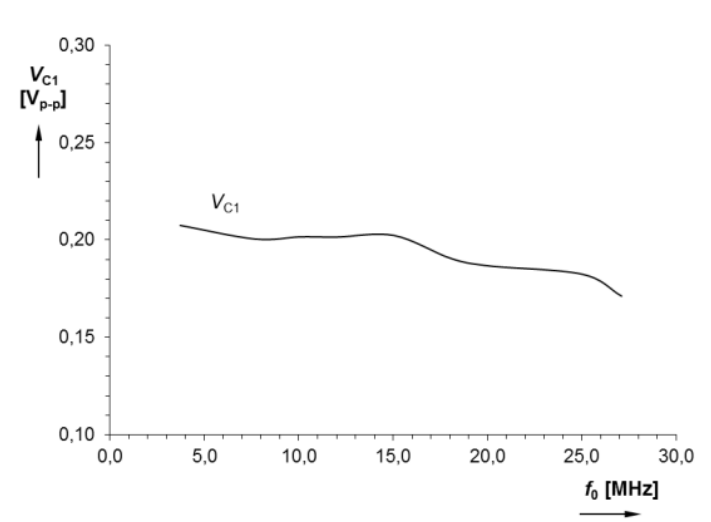

Fig.8. Dependence of output voltage on FO.

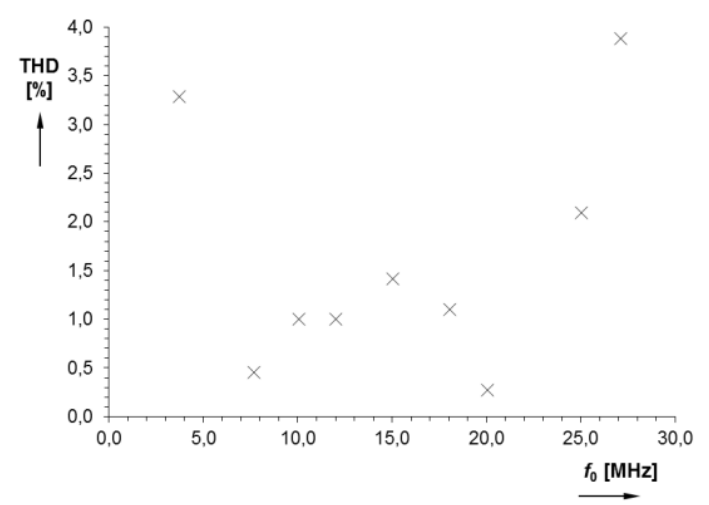

Fig.9. Dependence of THD on FO.

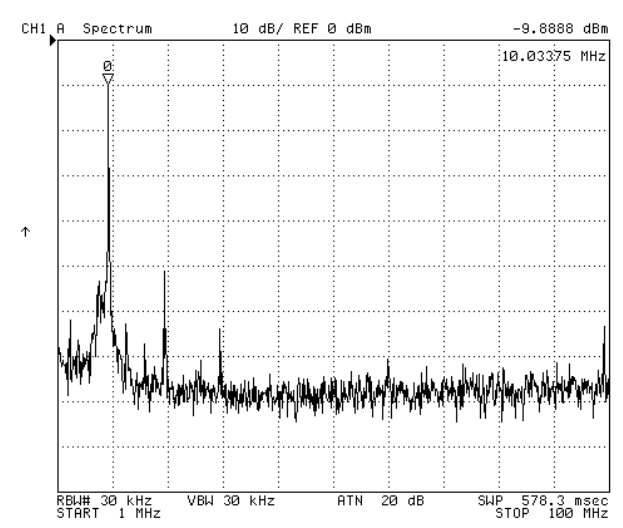

a)

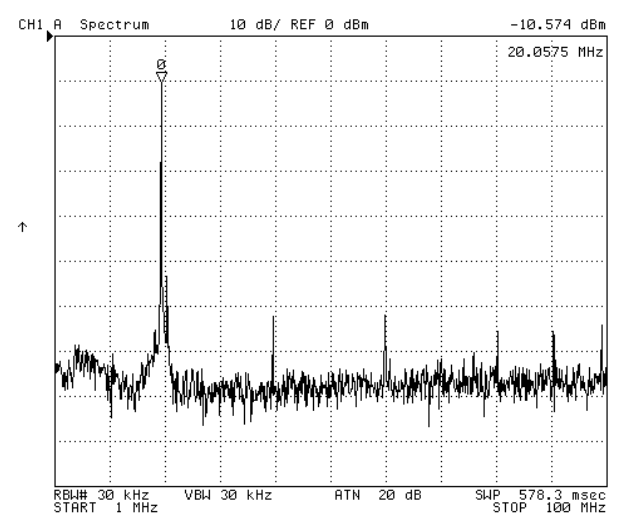

b)

Fig.10. Frequency spectrum for two selected frequencies: a) $10 \mathrm{MHz}$, b) $20 \mathrm{MHz}$
Fig.8. shows the dependence of output voltage $\left(V_{\mathrm{C} 1}\right)$ on FO and Fig.9. indicates total harmonic distortion (THD), both in the observed range of FO. Higher THD (above $1 \%$ ) is given by low and limited dynamical range of AD835 [25] and nonlinearity (DC transfer characteristic) of active device itself (second and third harmonic components at the output of AD835 are suppressed by $40 \mathrm{~dB}$ only for very high frequencies).

Exemplary spectrum analysis results are shown in Fig.10. for two selected frequencies of oscillation: $10 \mathrm{MHz}$ $\left(V_{\mathrm{SETA}}=-0.16 \mathrm{~V}, A=-0.34\right)$ and $20 \mathrm{MHz}\left(V_{\mathrm{SETA}}=1.74 \mathrm{~V}\right.$, $A=3.71)$.

\section{CONCLUSION}

Proposed oscillator offers interesting features for highfrequency design despite the lack of multiphase outputs. However, this feature is not commonly available in case of very simple circuits utilizing only basic elements (with respect to many still hypothetical structures [30]). Output level slightly fluctuates but change of output peak-to-peak voltage in observed range of FO $(3.7-27.1 \mathrm{MHz})$ is maximally $30 \mathrm{mV}$. THD varies between $0.2-3.7 \%$. Higher values are given especially by internal nonlinearity of AD835 (indicated in datasheet [25]), inappropriate time constant of AGC that is insufficient for very low and high FO (corners of tunability range) and achievement of limits of gain slope $B$ (EL2082), see Fig.7. THD achieving maximally $1.5 \%$ is allowed in narrower range $(7-20 \mathrm{MHz})$ of FO change. Both polarities of DC control voltage $V_{\text {SETA }}$ (utilization of voltage multiplier) allow important extension of FO control in comparison to single polarity control of gain $A$ (only FO range from 12 to $27.1 \mathrm{MHz}$ allowed for single/positive polarity of $A$ ). Power consumption is quite high in discrete solution (hundreds of $\mathrm{mW}$ ).

Parasitic analysis of real influences in the circuit is highly required for working capacitors below $100 \mathrm{pF}$. Results show that estimation is quite problematic and minimal deviation of expected and real values (combined with fabrication tolerances of active and passive elements) can be very large. In conclusion we can see that commercially available devices are really able to overcome the border of $10 \mathrm{MHz}$. Therefore, we can utilize them sufficiently for behavioral modeling of complex devices and systems [31], [32] operating in these bands. High-frequency features predetermine the proposed circuit, for example, as an easily tunable signal source in short wave and medium wave transmitters with amplitude or frequency modulation or digital amplitude keying.

\section{ACKNOWLEDGMENT}

Research described in the paper was supported by Czech Science Foundation project under No. 14-24186P.

\section{REFERENCES}

[1] Soliman, A.M. (1998). Novel oscillators using current and voltage followers. Journal of the Franklin Institute, 335 (6), 997-1007. 
[2] Keskin, A.U., Aydin, C., Hancioglu, E., Acar, C. (2006). Quadrature oscillator using current differencing buffered amplifiers (CDBA). Frequenz, 60 (6), 21-23.

[3] Sotner, R., Sevcik, B., Slezak, J., Petrzela, J., Brancik, L. (2011). Sinusoidal oscillator based on adjustable current amplifier and diamond transistors with buffers. Przeglad Elektrotechniczny, 87 (1), 266-270.

[4] Sotner, R., Hrubos, Z., Slezak, J., Dostal, T. (2010). Simply adjustable sinusoidal oscillator based on negative three-port current conveyors. Radioengineering, 19 (3), 446-453.

[5] Sotner, R., Jerabek, J., Petrzela, J., Dostal, T., Vrba, K. (2009). Electronically tunable simple oscillator based on single-output and multiple output transconductor. IEICE Electronics Express, 6 (20), 1476-1482.

[6] Herencsar, N., Minaei, S., Koton, J., Yuce, E., Vrba, K. (2013). New resistorless and electronically tunable realization of dual-output VM all-pass filter using VDIBA. Analog Integrated Circuits and Signal Processing, 74 (1), 141-154.

[7] Jaikla, W., Siripruchyanun, M., Bajer, J., Biolek, D. (2008). A simple current-mode quadrature oscillator using single CDTA. Radioengineering, 17 (4), 33-40.

[8] Pandey, N., Paul, S.K. (2011). Single CDTA-based current mode all-pass filter and its applications. Journal of Electrical and Computer Engineering, 2011, art. ID 897631.

[9] Songkla, S.N., Jaikla, W. (2012). Realization of electronically tunable current-mode first-order allpass filter and its application. International Journal of Electronics and Electrical Engineering, 2012 (6), 4043.

[10] Keawon, R., Jaikla, W. (2011) A resistor-less currentmode quadrature sinusoidal oscillator employing single CCCDTA and grounded capacitors. Przeglad Elektrotechniczny, 87 (8), 138-141.

[11] Senani, R. (1989). New electronically tunable OTA-C sinusoidal oscillator. Electronics Letters, 25 (4), 286287.

[12] Li, Y. (2010). Electronically tunable current-mode quadrature oscillator using single MCDTA. Radioengineering, 19 (4), 667-671.

[13] Sotner, R., Hrubos, Z., Herencsar, N., Jerabek, J., Dostal, T., Vrba, K. (2014). Precise electronically adjustable oscillator suitable for quadrature signal generation employing active elements with current and voltage gain control. Circuits Systems and Signal Processing, 33 (1), 1-35.

[14] Linares-Barranco, B., Rodriguez-Vazquez, A., Sanchez-Sinencio, E., Huertas, J.L. (1991). CMOS OTA-C high-frequency sinusoidal oscillators. IEEE Journal of Solid-State Circuits, 26 (2), 160-165.

[15] Prommee, P., Dejhan, K. (2002). An integrable electronic-controlled quadrature sinusoidal oscillator using CMOS operational transconductance amplifier. International Journal of Electronics, 89 (5), 365-379.

[16] Horng, J-W. (2009). Current-mode third-order quadrature oscillator using CDTAs. Active and Passive Electronic Components, 2009, art. ID 789171.
[17] Chatuverdi, B., Maheshwari, S. (2013). Third-order quadrature oscillators circuit with current and voltage outputs. ISRN Electronics, 2013, art. ID 385062.

[18] Surakampontorn, W., Thitimajshima, W. (1988). Integrable electronically tunable current conveyors. IEE Proceedings-G, 135 (2), 71-77.

[19] Fabre, A., Mimeche, N. (1994). Class A/AB secondgeneration current conveyor with controlled current gain. Electronics Letters, 30 (16), 1267-1268.

[20] Minaei, S., Sayin, O.K., Kuntman, H. (2006). A new CMOS electronically tunable current conveyor and its application to current-mode filters. IEEE Transaction on Circuits and Systems - I, 53 (7), 1448-1457.

[21] Sedra, A., Smith, K.C. (1970). A second generation current conveyor and its applications. IEEE Transaction on Circuit Theory, CT-17 (2), 132-134.

[22] Svoboda, J.A., McGory, L., Webb, S. (1991). Applications of a commercially available current conveyor. International Journal of Electronics, 70 (1), 159-164.

[23] Texas Instruments. VCA810: High gain adjust range, wideband and variable gain amplifier. http://www. ti.com/lit/ds/symlink/vca810.pdf.

[24] Texas Instruments. LMH6505: Wideband, low power, linear-in- $d B$, variable gain amplifier. http:// www.ti.com/lit/ds/symlink/lmh6505.pdf.

[25] Analog Devices. AD835: $250 \mathrm{MHz}$, voltage output 4quadrant multiplier. http://www.analog.com/static/ imported-files/data_sheets/AD835.pdf.

[26] Sotner, R., Jerabek, J., Petrzela, J., Herencsar, N., Prokop, R., Vrba, K. (2014). Second-order simple multiphase oscillator using Z-copy controlled-gain voltage differencing current conveyor. Elektronika ir Elektrotechnika, 20 (9), 13-18.

[27] Sotner, R., Jerabek, J., Herencsar, N., Horng, J-W., Vrba, K. (2015). Electronically linearly voltage controlled second-order harmonic oscillators with multiples of pi/4 phase shifts. In 38th International Conference on Telecommunications and Signal Processing (TSP), 9-11 July 2015. IEEE, 708-712.

[28] Intersil. EL2082: Current-mode multiplier. http:// www.intersil.com/data/fn/fn7152.pdf.

[29] Texas Instruments. OPA860: Wide-bandwidth, operational transconductance amplifier (OTA) and buffer. http://www.ti.com/lit/ds/symlink/opa860.pdf.

[30] Biolek, D., Senani, R., Biolkova, V., Kolka, Z. (2008). Active elements for analog signal processing: Classification, review, and new proposal. Radioengineering, 17 (4), 15-32.

[31] Sotner, R., Kartci, A., Jerabek, J., Herencsar, N., Dostal, T., Vrba, K. (2012). An additional approach to model current followers and amplifiers with electronically controllable parameters from commercially available ICs. Measurement Science Review, 12 (6), 255-265.

[32] Odon, A. (2010). Modelling and simulation of the pyroelectric detector using MATLAB/Simulink. Measurement Science Review, 10 (6), 195-199.

Received September 3, 2015. Accepted March 22, 2016. 